\title{
Loss of Fas expression and high expression of HLA-E promoting the immune escape of early colorectal cancer cells
}

\author{
RENXIANG HUANG ${ }^{1}$, DONGYANG ZHANG ${ }^{2}$, FENG LI $^{1}$, ZILI XIAO ${ }^{1}$, \\ MEILING WU ${ }^{2}$, DONGYUN SHI ${ }^{2}$, PING XIANG ${ }^{1}$ and ZHIJUN BAO ${ }^{1}$ \\ ${ }^{1}$ Digestive Endoscopy Center, Huadong Hospital Affiliated to Fudan University, Shanghai 200040; ${ }^{2}$ Department of \\ Biochemistry and Molecular Biology, School of Basic Medical Sciences, Fudan University, Shanghai 200032, P.R. China
}

Received February 11, 2016; Accepted December 20, 2016

DOI: $10.3892 / \mathrm{ol} .2017 .5891$

\begin{abstract}
Previous studies have investigated the mechanisms of immune evasion of tumor cells in numerous types of advanced solid malignant tumor, and several types of immune preparations have been administered as antitumor adjuvant therapies. However, in the majority of studies, the efficacy of therapies has been revealed to be limited. The present study aimed to investigate the immune evasion mechanisms employed by early colorectal cancer cells and the expression of the molecules associated with immune evasion during the malignant transformation process of normal colorectal epithelial cells to measure the effects of immune intervention for early colorectal cancer, and to improve the efficacy of immunotherapy. A total of 60 colorectal tissues, including 15 normal mucosa, 15 adenoma, 15 early cancer and 15 advanced cancer tissues, from patients undergoing endoscopic procedures in Huadong Hospital Affiliated to Fudan University (Shanghai, China) were collected. A comparison of baseline characteristics among these four groups was performed. The expression levels of human leukocyte antigen-A (HLA-A), apoptosis antigen 1 (Fas), c-c chemokine receptor type 5 (CCR5), Fas ligand (FasL) and HLA-E in each group were detected by immunohistochemical analysis. Furthermore, 15 patients with advanced colorectal cancer were enrolled into the present study. Advanced cancer and paracancer tissues (normal mucosal tissues $3 \mathrm{~cm}$ away from the margin of cancer tissues) were collected from each patient by colonoscopic biopsy. The expression levels of HLA-A, Fas, CCR5, FasL and HLA-E in each group were detected by western blot analysis. During the malignant transformation process of normal colorectal epithelial cells, the expression levels of CCR5, FasL and
\end{abstract}

Correspondence to: Professor Zhijun Bao, Digestive Endoscopy Center, Huadong Hospital Affiliated to Fudan University, 221 West Yan'an Road, Jing'an, Shanghai 200040, P.R. China

E-mail: xinyi8681@sina.com

Keywords: early colorectal cancer,immune escape, human leukocyte antigen-A, apoptosis antigen $1, \mathrm{C}-\mathrm{C}$ chemokine receptor type 5, apoptosis antigen 1 ligand, human leukocyte antigen class I histocompatibility antigen, alpha chain $\mathrm{E}$
HLA-E increased significantly $(\mathrm{P}<0.001)$, whilst the expression levels of Fas reduced significantly $(\mathrm{P}=0.0271)$. In the early cancer group, the expression levels of Fas reduced significantly $(\mathrm{P}=0.0239)$, whilst the expression levels of HLA-E increased significantly $(\mathrm{P}<0.001)$ compared with adenoma group. In conclusion, a loss of Fas expression and high expression levels of HLA-E may promote the immune evasion of early colorectal cancer cells.

\section{Introduction}

Colorectal cancer is a common type of malignant tumor of the digestive tract. In 2012, the morbidity rates of colorectal cancer in developed countries ranked third and second in the morbidity rates of malignant tumors in males and females, respectively (1). During this time, the morbidity rates of colorectal cancer also increased markedly in China. In 2013, the morbidity rates of colorectal cancer ranked fifth amongst the morbidity rates of all types of malignant tumors in China, whilst in the urban population of China, the morbidity rates of colorectal cancer were ranked second amongst the morbidity rates of all types of malignant tumors (2). As surgery is the most effective therapeutic method for colorectal cancer up to date, the primary treatment of colorectal cancer is surgery, supplemented by radiotherapy and chemotherapy. At present, along with the continued investigation of the pathogenesis of colorectal cancer, immunotherapy of colorectal cancer has become more acceptable and has a potentially wide application.

In 1909 Ehrlich first proposed the concept of immune surveillance (3). In 1959, Thomas proposed that a low expression of the tumor cell antigen or damaged cellular immune function was an important factor of tumorigenesis (3). Subsequently, Burnet developed the immune surveillance theory, which hypothesized that the immune system may perform surveillance, recognizing and eliminating novel antigens expressed, alien components or mutated cells, to maintain the stability of the internal environment (3). Although the immune system may generate an immune response to tumor cells and eliminate the tumor, there remains a certain proportion of primary tumors that may metastasize and recur. Thus, certain types of tumor may escape the immune system, which is termed tumor immune evasion. Previous studies have indicated that 
the immune evasion mechanism of colorectal cancer cells may be separated into 3 major categories: Low immunogenicity of colorectal cancer cells, due to a loss or low expression of classical human leukocyte antigen (HLA) molecules $(4,5)$ and a loss of apoptosis antigen 1 (Fas) expression (6,7); dysfunction of the effector cells due to high expression levels of the c-c chemokine receptor type 5 (CCR5) from tumor infiltrating regulatory T-cells (8) and the expression of immunosuppressive factors by colorectal cancer cells due to high expression levels of the Fas ligand (FasL) (9-11); and HLA class I histocompatibility antigen, $\alpha$ chain E (HLA-E) as a type of nonclassical HLAImolecule $(12,13)$.

At present, studies have revealed that the immune evasion mechanisms of tumor cells in certain types of advanced solid malignant tumors, and several types of immune preparations have been applied as antitumor adjuvant therapy (3). However, in the majority of studies, the efficacy is limited, which may be associated with the different immune microenviroments between advanced and early cancers. Thus, an investigation of the immune evasion mechanisms of early colorectal cancer cells and a trial of an immune-based intervention for early colorectal cancer may generate data to improve the efficacy of immunotherapy in this type of cancer.

To date, previous studies on the immune evasion mechanisms of early colorectal cancer have not been performed. The present study aimed to investigate the immune evasion mechanisms in early colorectal cancer cells and the expression of immune evasion-associated molecules during the malignant transformation process of normal colorectal epithelial cells.

\section{Materials and methods}

Tissue samples. All tissue specimens were collected between June 2014 and June 2015. A total of 45 colorectal specimens, including 15 adenoma, 15 early cancer and 15 advanced cancer tissues, from patients undergoing endoscopic procedures such as endoscopic submucosal dissection, endoscopic mucosal resection, polypectomy and tumor biopsy in the Huadong Hospital Affiliated to Fudan University (Shanghai, China) were collected randomly. A total of 15 adenoma tissues were tubulovillous adenomas, as confirmed by pathology. Amongst the 15 patients with early cancer tissues, 13 were high grade intraepithelial neoplasia and 2 were intramucosal cancer. In the patients with advanced cancer, 2 were Dukes A stage, 4 were Dukes B stage, 5 were Dukes C1 stage and 4 were Dukes D stage (14). For a control group, 15 normal colorectal mucosa specimens from 15 consecutive healthy physical examination persons were collected by colonoscopic biopsy. All 60 specimens were divided into four groups according to the type of tissue. All patients and controls enrolled provided informed consent. The present study was approved by Ethics Committee of Huadong Hospital (Shanghai, China; trial registration no. ChiCTR-OOC-15007390).

Immunohistochemical analysis. The formalin-fixed and paraffin-embedded tissue specimens were assessed by immunohistochemical analysis using the DouSPTM IHC kit (Fuzhou Maixin Biotech Co., Ltd., Fuzhou, China), according to the protocol of the manufacturer. Anti-HLA-A (cat. no. 15240-1-AP) and anti-Fas (cat. no. 13098-1-AP) antibodies were purchased from Wuhan Sanying Biotechnology (Wuhan, China). Anti-CCR5 (cat. no. ab110103) antibody was purchased from Abcam (Cambridge, MA, USA). Anti-FasL (cat. no. ARG 53012) and anti-HLA-E (cat. no. ARG 63017) antibodies were purchased from Arigo (Taiwan, China). Specimens were incubated with the primary antibody at $4{ }^{\circ} \mathrm{C}$ overnight. The secondary antibodies (cat. no. 7074) were purchased from Cell Signaling Technology (Danvers, MA, USA). Specimens were incubated with the secondary antibody at room temperature for $2 \mathrm{~h}$. The secondary antibodies were labeled and visualized using a color solution (Tiangen Biotech Co., Ltd., Beijing, China). All the dilutions were 1:100. The expression levels of all the proteins were determined using a semiquantitative method as previously described (11) and assessed as strong positive, positive-staining cells $\geq 50 \%$; weak positive, positive-staining cells $\geq 25$ but $<50 \%$, and negative, lack of staining or positive-staining cells $<25 \%$. Positive-staining reactions were measured under a light microscope using x400 magnification.

Western blot analysis. The expression levels of HLA-A, Fas, CCR5, FasL and HLA-E in each group were detected by western blot analysis. As early colorectal cancer lesions were often small and the majority of such lesions generated from adenoma, the present study did not obtain fresh colorectal adenoma and early cancer tissues for western blot analysis to avoiding ethical issues.

A total of 15 patients with advanced colorectal cancer were enrolled into the present study. Advanced cancer and paracancer tissues (normal mucosal tissues $3 \mathrm{~cm}$ away from the margin of cancer tissues) were collected from each patient by colonoscopic biopsy. The specimens were divided into two groups according to the type of tissue. In the 15 patients with advanced colorectal cancer, 3 were Dukes A stage, 5 were Dukes B stage, 4 were Dukes C1 stage and 3 were Dukes C2 stage. By location, 4 were in the ascending colon, 1 was in the transverse colon, 1 was in the descending colon, 5 were in the sigmoid and 4 were in rectum.

Tissue samples from the patients were extracted using loading buffer (0.05 M Tris, 10\% glycerol, 4\% $\beta$-mercaptoethanol, $2 \%$ SDS, $0.1 \%$ bromophenol blue, $\mathrm{pH}$ 6.8) with $1 \mathrm{mM}$ phenylmethylsulfonyl fluoride (Beyotime Institute of Biotechnology, Haimen, China), separated by SDS-PAGE (20 $\mu \mathrm{g}$ protein per lane) on a $12 \%$ gel and transferred to polyvinylidene fluoride membranes. The Tanon-5200 Chemi-luminescent Imaging System (Tanon Science and Technology Co., Ltd., Shanghai, China) was used to observe the levels of protein expression. The primary antibodies and secondary antibodies were used as described in the aforementioned immunohistochemical analysis protocol.

Statistical analysis. All analyses were performed using Statistical Analysis System 9.13 statistical software package (SAS Institute, Inc., Cary, NA, USA). The normal distribution of the measurement data was evaluated by unpaired Student's t-test or analysis of variance. The enumeration data was evaluated by a $\chi^{2}$ test, and Fisher exact test was used if the frequency was low. $\mathrm{P}<0.05$ was considered to indicate a statistically significant difference. 
Table I. Baseline characteristics of patients with colorectal cancer.

\begin{tabular}{lccccr}
\hline & \multicolumn{5}{c}{ Value } \\
\cline { 2 - 4 } Characteristic & $\begin{array}{c}\text { Normal mucosa } \\
(\mathrm{n}=15)\end{array}$ & $\begin{array}{c}\text { Adenoma } \\
(\mathrm{n}=15)\end{array}$ & $\begin{array}{c}\text { Early cancer } \\
(\mathrm{n}=15)\end{array}$ & $\begin{array}{c}\text { Advanced cancer } \\
(\mathrm{n}=15)\end{array}$ & P-value \\
\hline Gender (male/female) & $9 / 6$ & $7 / 8$ & $11 / 4$ & $10 / 5$ & 0.481 \\
Age, years & $58.82 \pm 6.82$ & $63.47 \pm 6.38$ & $62.20 \pm 9.06$ & $68.54 \pm 11.53$ & 0.060 \\
Location & 3 & & & 2 & 0.081 \\
Ascending colon & 4 & 1 & 1 & 0 & \\
Transverse colon & 2 & 0 & 3 & 4 & \\
Descending colon & 3 & 4 & 5 & 8 \\
Sigmoid & 3 & 2 & 4 & & \\
Rectum & 3 & & & & \\
\hline
\end{tabular}

\section{Results}

Baseline characteristics. The gender, age and sample location of patients between the four groups were compared and presented in Table I.

Immunohistochemical analysis. The expression levels of HLA-A, Fas, CCR5, FasL and HLA-E were analyzed between the four groups: Normal mucosa, adenoma, early cancer group and advanced cancer groups. It was observed that the expression levels of HLA-A demonstrated no significant difference between cancer stages during the malignant transformation process of normal colorectal epithelial cells (Fig. 1; Table II). The expression levels of Fas reduced significantly along with an increased malignancy of the epithelial cells $(\mathrm{P}=0.0271)$. In addition, the expression of Fas reduced significantly in the early cancer group $(\mathrm{P}=0.0239)$ compared with adenoma group, as demonstrated in Fig. 2 and Table II. The expression of CCR5 and FasL increased significantly with increased malignancy of the epithelial cells $(\mathrm{P}<0.001)$. However, no significant difference in the expression levels of CCR5 and FasL was observed between the early cancer and adenoma groups ( $\mathrm{P}>0.05)$, as demonstrated in Figs. 3 and 4, and Table II. The expression of HLA-E increased significantly as the malignancy of the epithelial cells increased $(\mathrm{P}<0.001)$. In addition, the expression levels of HLA-E increased significantly in the early cancer group $(\mathrm{P}<0.001)$ compared with adenoma group, as illustrated in Fig. 5 and Table II.

Western blot analysis. The expression of HLA-A, Fas, CCR5, FasL and HLA-E in the advanced colorectal cancer and paracancer tissues were analyzed. The results of the western blot analysis were consistent with the results of the immunohistochemical analysis. No significant difference was observed in the expression levels of HLA-A between the advanced colorectal cancer and paracancer groups, as demonstrated in Figs. 6 and 11, the expression of Fas reduced significantly in the advanced colorectal cancer group compared with paracancer group, as illustrated in Figs. 7 and 11, whilst the expression levels of CCR5, FasL and HLA-E increased significantly in the advanced colorectal cancer group compared with paracancer group, as demonstrated in Figs. 8-11.

\section{Discussion}

The immune system can perform surveillance, recognizing and eliminating newly-expressed antigens, alien components or mutated cells. However, some tumors may escape attack from immune system, which is termed immune evasion. Previous studies have reported that the immune evasion mechanisms of colorectal cancer cells may include a low immunogenicity of colorectal cancer cells, dysfunction of effector cells and the expression of immunosuppressive factors by colorectal cancer cells $(4,6,8,11,12)$. The present study aimed to investigate the immune evasion mechanisms in early colorectal cancer cells and the expression of immune evasion-associated molecules during the malignant transformation process of normal colorectal epithelial cells, to measure the effects of immune intervention for early colorectal cancer and to generate data to improve the efficacy of immunotherapy for this type of cancer.

The present study indicated that HLA-A was highly expressed in normal colorectal mucosa, colorectal adenoma, early colorectal cancer and advanced colorectal cancer tissues, and that no significant difference was observed between the 4 groups $(\mathrm{P}>0.05)$. According to previous studies, the association between the expression of HLA class I molecules and the immune evasion of colorectal cancer cells remains controversial. Hypothetically, tumor antigens may be presented on the cell surface by HLA class I molecules and be recognized by cytotoxic T lymphocytes (CTL), which may kill tumor cells (3). Conversely, if the expression of HLA class I molecules is lost in tumor cells, tumor cells may be destroyed by natural killer (NK) cell-mediated cytotoxicity. Bernal et al (4) hypothesized that $\beta 2$-microglobulin is an important component of major histocompatibility complex (MHC) class I molecules. There is evidence to demonstrate that a loss of $\beta 2$-microglobulin may contribute to tumor immune evasion, particularly in colorectal cancer and melanoma. An additional study demonstrated that damaging the $\beta 2$-microglobulin gene, located at chromosome 15, may result in a loss of MHC class I molecule expression and tumor immune evasion (4). As illustrated by 
Table II. Immunohistochemical analysis: The expression of HLA-A, Fas, CCR5, FasL and HLA-E among the four groups.

\begin{tabular}{|c|c|c|c|c|c|c|}
\hline \multirow[b]{2}{*}{ Protein expression } & \multicolumn{4}{|c|}{ No. of patients } & \multirow[b]{2}{*}{ P-value } & \multirow[b]{2}{*}{$\mathrm{P}^{\prime}$-value } \\
\hline & $\begin{array}{l}\text { Normal mucosa } \\
\qquad(\mathrm{n}=15)\end{array}$ & $\begin{array}{l}\text { Adenoma } \\
(\mathrm{n}=15)\end{array}$ & $\begin{array}{l}\text { Early cancer } \\
\quad(\mathrm{n}=15)\end{array}$ & $\begin{array}{l}\text { Advanced cancer } \\
\quad(\mathrm{n}=15)\end{array}$ & & \\
\hline HLA-A & & & & & 0.460 & 0.682 \\
\hline Negative & 0 & 0 & 2 & 1 & & \\
\hline Weak & 2 & 4 & 3 & 1 & & \\
\hline Strong & 13 & 11 & 10 & 13 & & \\
\hline Fas & & & & & 0.0271 & 0.0239 \\
\hline Negative & 3 & 1 & 8 & 8 & & \\
\hline Weak & 4 & 7 & 3 & 5 & & \\
\hline Strong & 8 & 7 & 4 & 2 & & \\
\hline CCR5 & & & & & $<0.001$ & 0.176 \\
\hline Negative & 14 & 7 & 2 & 2 & & \\
\hline Weak & 1 & 3 & 5 & 5 & & \\
\hline Strong & 0 & 5 & 8 & 8 & & \\
\hline FasL & & & & & $<0.001$ & 0.605 \\
\hline Negative & 15 & 10 & 8 & 4 & & \\
\hline Weak & 0 & 5 & 5 & 8 & & \\
\hline Strong & 0 & 0 & 2 & 3 & & \\
\hline HLA-E & & & & & $<0.001$ & $<0.001$ \\
\hline Negative & 15 & 15 & 5 & 3 & & \\
\hline Weak & 0 & 0 & 9 & 6 & & \\
\hline Strong & 0 & 0 & 1 & 6 & & \\
\hline
\end{tabular}

The P-value is comparison amongst 4 groups, $\mathrm{P}^{\prime}$ adenoma group vs. early cancer group. HLA-A, human leukocyte antigen-A; Fas, apoptosis antigen 1; CCR5, C-C chemokine receptor type 5; FasL, apoptosis antigen 1 ligand; HLA-E, human leukocyte antigen class I histocompatibility antigen, alpha chain E.
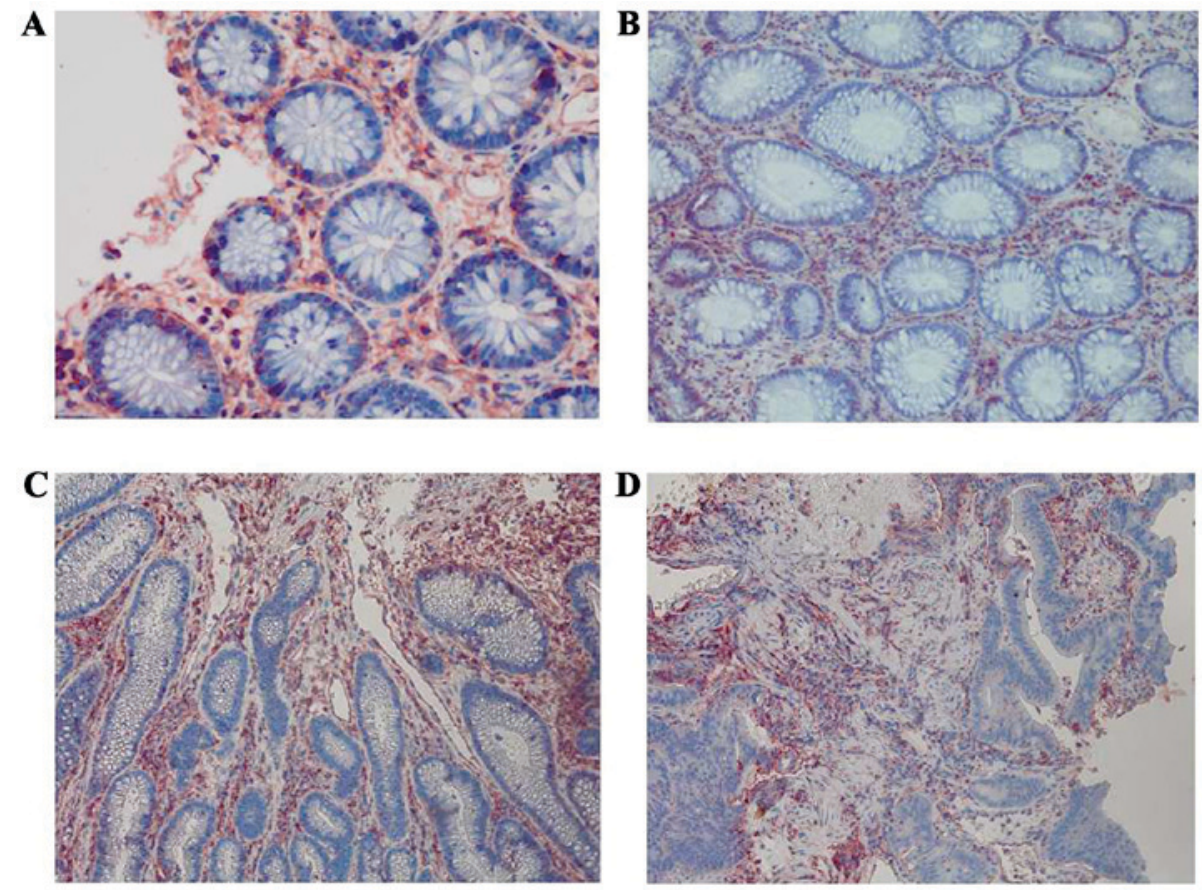

Figure 1. Immunohistochemical staining of HLA-A among the four groups at a magnification of $\mathrm{x} 400$. (A) Strong positive expression of HLA-A in normal colorectal mucosa. (B) Strong positive expression of HLA-A in colorectal adenoma. (C) Strong positive expression of HLA-A in early colorectal cancer. (D) Strong positive expression of HLA-A in advanced colorectal cancer. HLA-A, human leukocyte antigen-A. 
$\mathbf{A}$
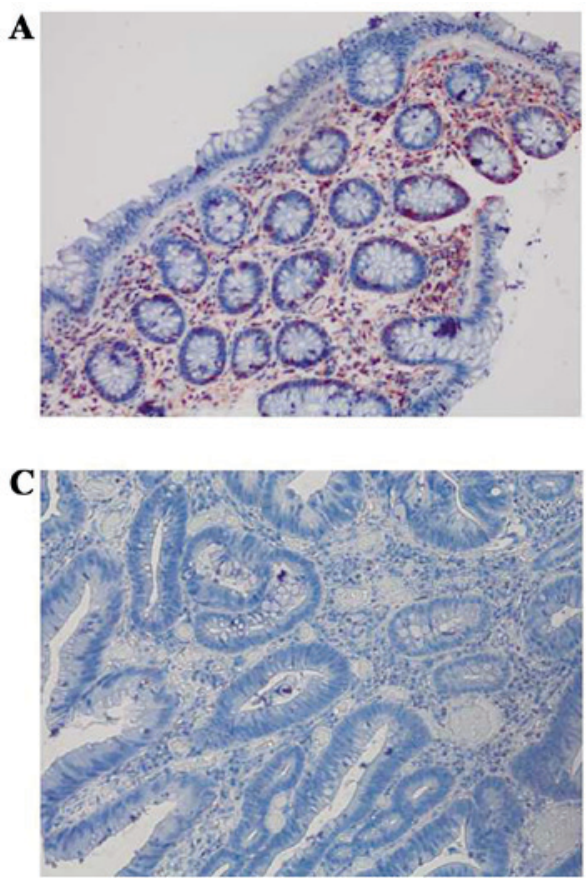
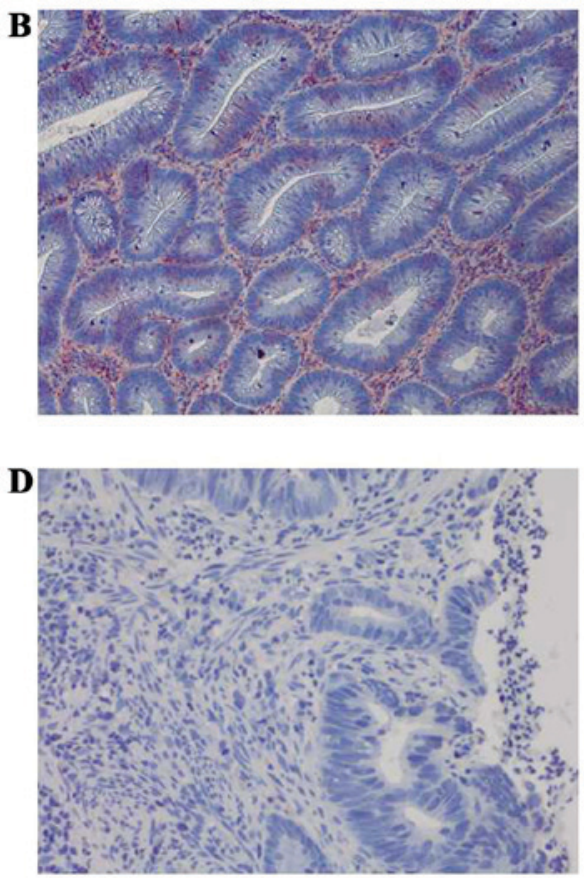

Figure 2. Immunohistochemical staining of Fas among the four groups at a magnification of x400. (A) Strong positive expression of Fas in normal colorectal mucosa. (B) Strong positive expression of Fas in colorectal adenoma. (C) Negative expression of Fas in early colorectal cancer. (D) Negative expression of Fas in advanced colorectal cancer. Fas, apoptosis antigen 1.
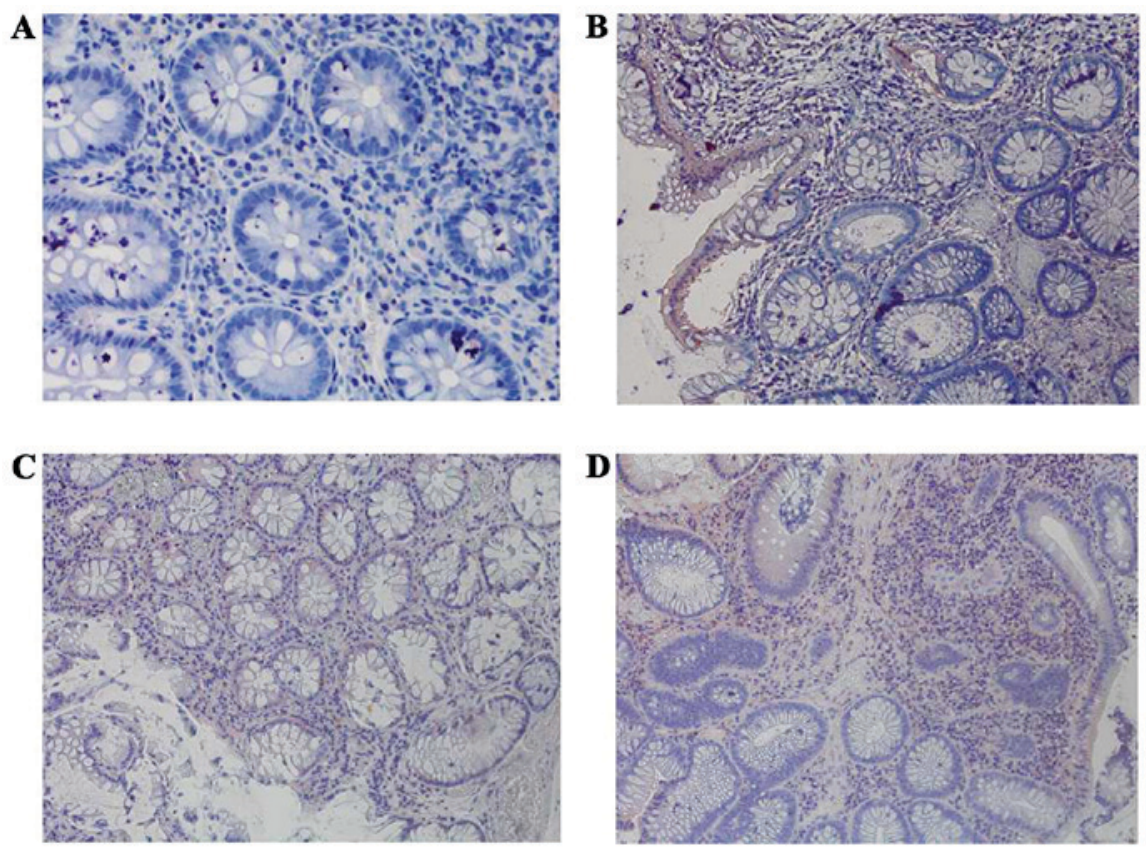

Figure 3. Immunohistochemical staining of CCR5 among the four groups at a magnification of x400. (A) Negative expression of CCR5 in normal colorectal mucosa. (B) Weak positive expression of CCR5 in colorectal adenoma. (C) Weak positive expression of CCR5 in early colorectal cancer. (D) Strong positive expression of CCR5 in advanced colorectal cancer. CCR5, C-C chemokine receptor type 5.

Sandel et al (15), MHC class I molecules were not expressed in $72 \%$ of 88 colorectal cancer specimens, whilst MHC class I molecules were expressed normally in $28 \%$ specimens. However, NK cells were distributed sparsely in the epithelium of the primary tumor, where $\mathrm{CD} 8^{+} \mathrm{T}$ cells were distributed densely instead, indicating that the immune microenviroment was disordered in primary colorectal cancer. Conversely, Menon et al (16) revealed that HLA-A was expressed in up to
98\% of 82 primary colorectal cancer specimens. Other studies demonstrated that the downregulated expression of HLA class I molecules was deleterious to the prognosis of patients with colorectal cancer $(5,17,18)$. A possible explanation is that if the expression of HLA class I molecules is completely eliminated in tumor cells, the tumor cells may be destroyed by NK cell-mediated cytotoxicity, whilst a downregulated expression of HLA class I antigens may protect tumor cells from immune 

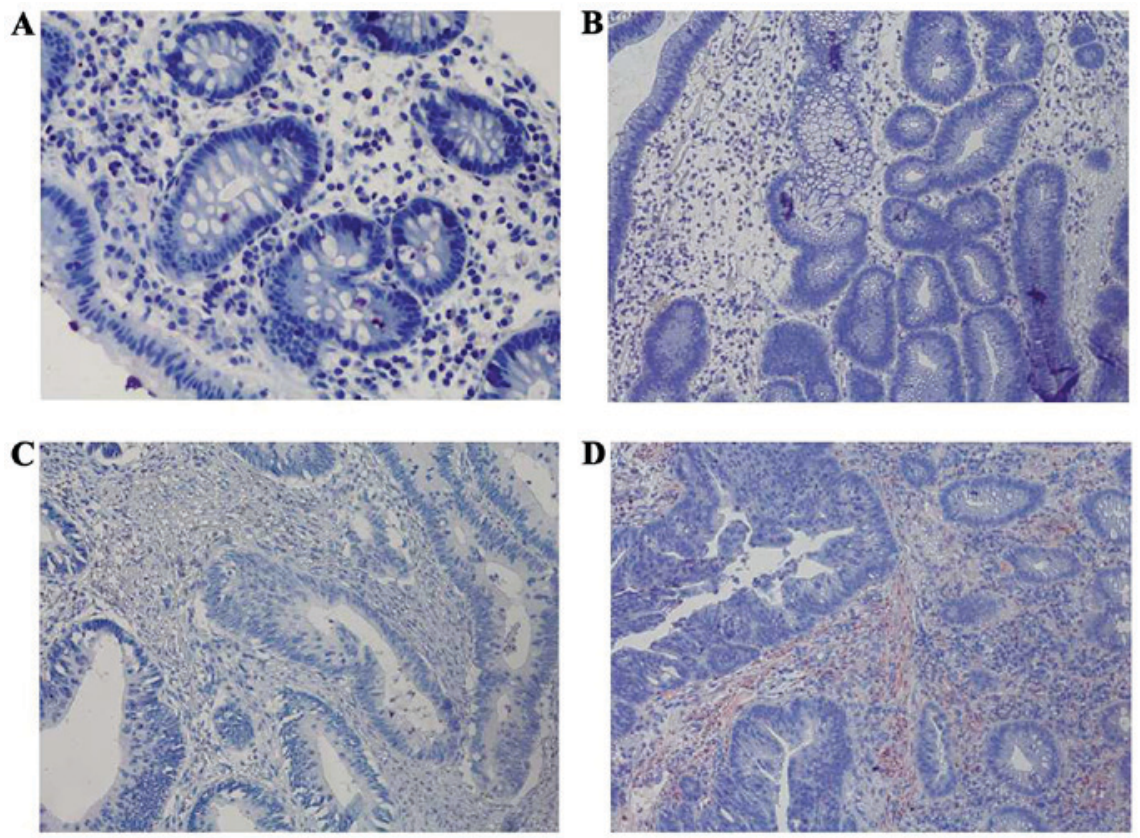

Figure 4. Immunohistochemical staining of FasL among the four groups at a magnification of x400. (A) Negative expression of FasL in normal colorectal mucosa. (B) Negative expression of FasL in colorectal adenoma. (C) Negative expression of FasL in early colorectal cancer. (D) Strong positive expression of FasL in advanced colorectal cancer. FasL, apoptosis antigen 1 ligand.
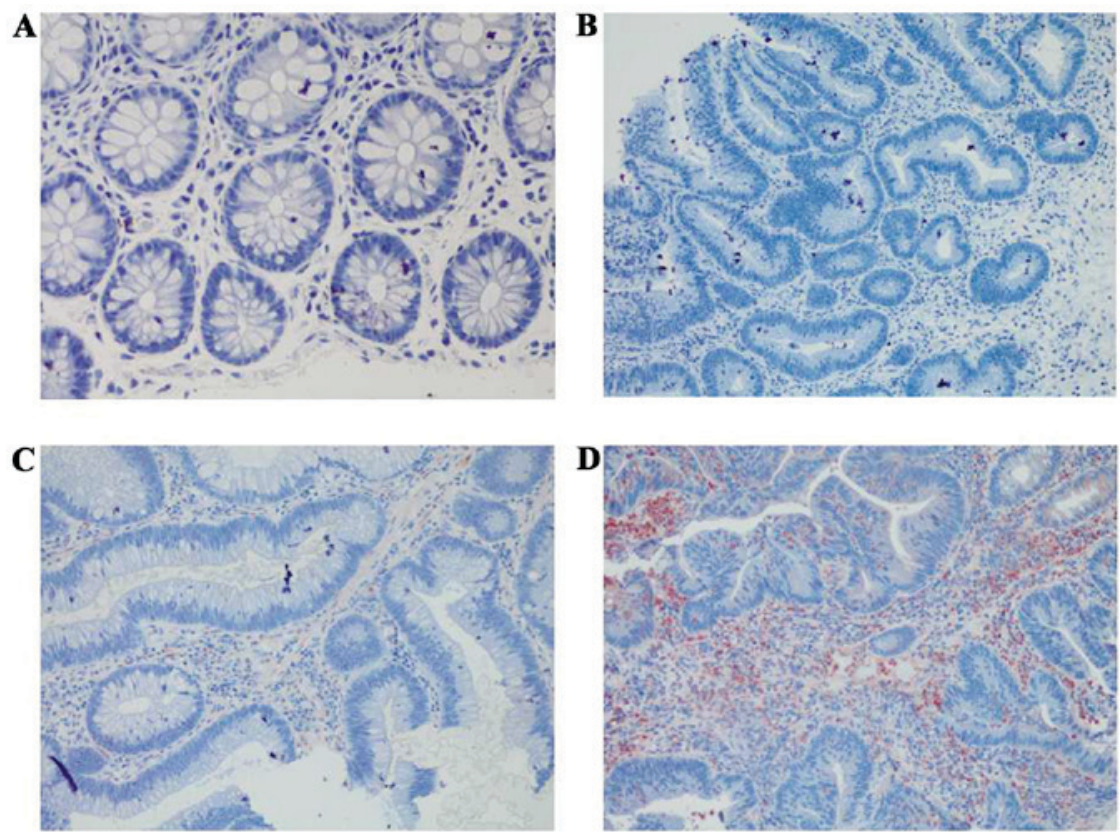

Figure 5. Immunohistochemical staining of HLA-E among the four groups at a magnification of $x 400$. (A) Negative expression of HLA-E in normal colorectal mucosa. (B) Negative expression of HLA-E in colorectal adenoma. (C) Weak positive expression of HLA-E in early colorectal cancer. (D) Strong positive expression of HLA-E in advanced colorectal cancer. HLA-E, human leukocyte antigen class I histocompatibility antigen, alpha chain E.

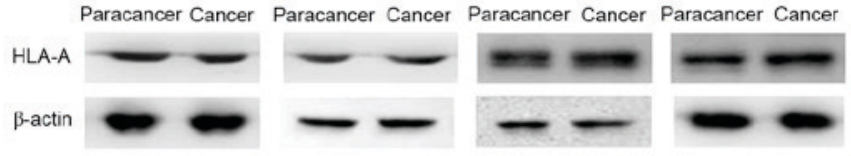

Figure 6. Expression of HLA-A in paracancer tissues and advanced colorectal cancer tissues. No significant difference was observed in the expression levels of HLA-A in the advanced colorectal cancer group compared with the paracancer groups. Staging and location of samples from left to right: Dukes A, ascending colon; Dukes C1, ascending colon; Dukes C1, rectum; and Dukes C1, sigmoid $\beta$-actin was the internal reference. HLA-A, human leukocyte antigen-A. attack by T cells and NK cells $(5,17,18)$. In summary, although studies investigating the association between the expression of HLA class I molecules and the immune evasion of colorectal cancer cells are common, the conclusions are different. In the present study, HLA-A was highly expressed in all 4 groups. Thus, investigating the precise mechanism of HLA-A and immune evasion of colorectal cancer cells may rely on additional studies of molecular transduction mechanisms, with larger sample sizes. 


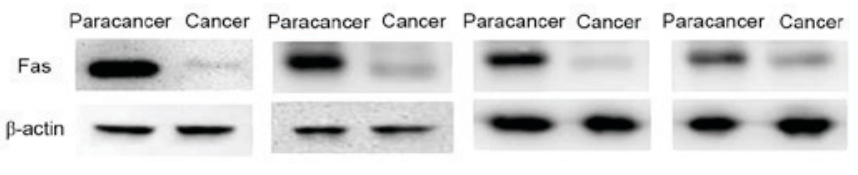

Figure 7. Expression of Fas in paracancer tissues and advanced colorectal cancer tissues. The expression of Fas was decreased in the advanced colorectal cancer group compared with the paracancer group. Staging and location of samples from left to right: Dukes C1, ascending colon; Dukes C1, rectum; Dukes $\mathrm{C} 1$, sigmoid; and Dukes B, rectum. $\beta$-actin was the internal reference. Fas, apoptosis antigen 1.

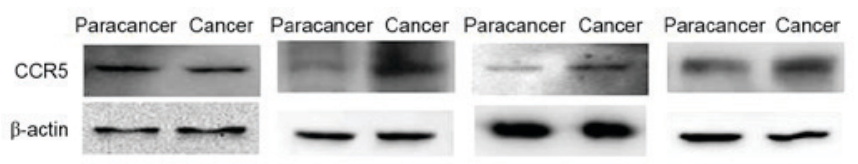

Figure 8. Expression of CCR5 in paracancer tissues and advanced colorectal cancer tissues. The expression of CCR5 increased to certain extent in the advanced colorectal cancer group compared with the paracancer group. Staging and location of samples from left to right: Dukes C2, sigmoid; Dukes $\mathrm{C} 1$, ascending colon; Dukes $\mathrm{C} 1$, sigmoid; and Dukes B, rectum. $\beta$-actin was the internal reference. CCR, C-C chemokine receptor type 5.

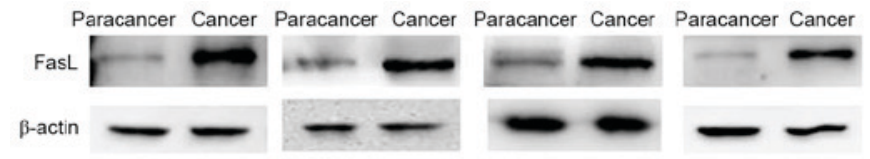

Figure 9. Expression of FasL in paracancer tissues and advanced colorectal cancer tissues. The expression of FasL increased in the advanced colorectal cancer group compared with the paracancer group. Staging and location of samples from left to right: Dukes C1, ascending colon; Dukes C1, rectum; Dukes $\mathrm{C} 1$, sigmoid; and Dukes B, rectum. $\beta$-actin was the internal reference. FasL, apoptosis antigen 1 ligand.

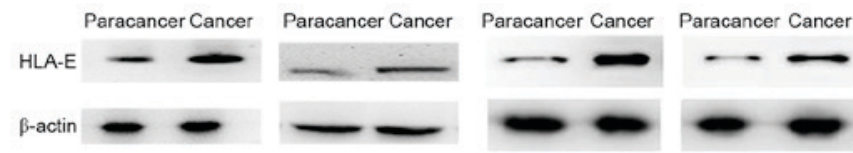

Figure 10. Expression of HLA-E in paracancer tissues and advanced colorectal cancer tissues. The expression of HLA-E increased in the advanced colorectal cancer group compared with the paracancer group. Staging and location of samples from left to right: Dukes C1, rectum; Dukes B, sigmoid; Dukes C1, sigmoid; and Dukes B, rectum. $\beta$-actin was the internal reference. HLA-E, human leukocyte antigen class I histocompatibility antigen, alpha chain E.
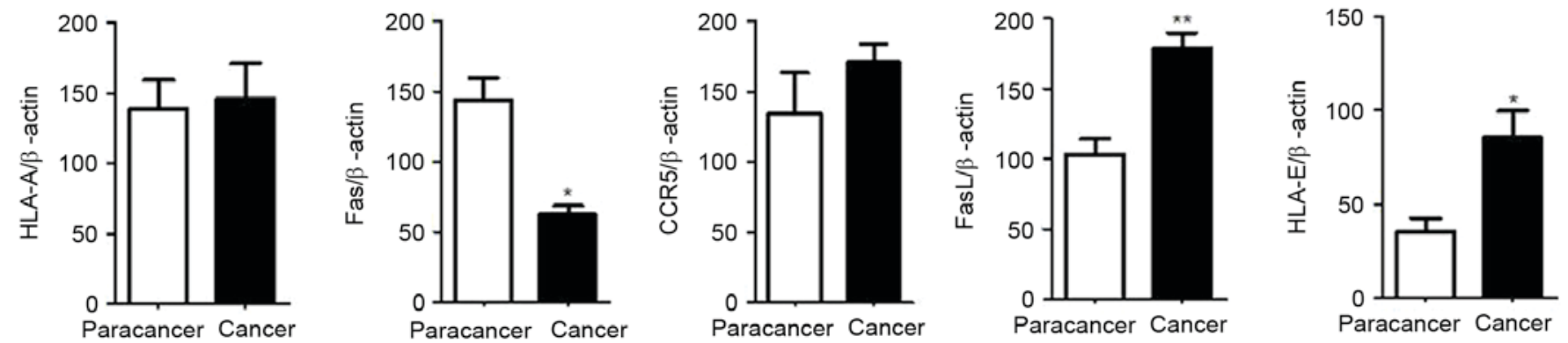

Figure 11. Western blot analysis gray value comparison of HLA-A, Fas, CCR5, FasL and HLA-E. $\beta$-actin was the internal reference. Results were represented as the mean \pm standard deviation. Paired $t$ test was used for statistical analysis. ${ }^{*} \mathrm{P}<0.05 ;{ }^{* *} \mathrm{P}<0.01$. HLA-A, human leukocyte antigen-A; Fas, apoptosis antigen 1; CCR5, C-C chemokine receptor type 5; FasL, apoptosis antigen 1 ligand; HLA-E, human leukocyte antigen class I histocompatibility antigen, alpha chain E.

Previous studies have demonstrated that Fas is highly expressed in normal colorectal epithelial cells, whilst a loss of Fas expression and anti-Fas-mediated apoptosis in colorectal cancer cells contributed to tumor invasion and metastasis $(6,7,19)$. The present study indicated that the expression of Fas reduced significantly as the malignancy of the epithelial cells increased $(\mathrm{P}=0.0271)$. Furthermore, the expression of Fas reduced significantly in early cancer group $\left(\mathrm{P}^{\prime}=0.0239\right)$ compared with adenoma group, which suggested that a loss of Fas expression may serve an important role in the immune evasion in early colorectal cancer.

Chang et al (8) revealed that CCR5 was highly expressed in tumor infiltrating cluster of differentiation (CD) $4^{+}$forkhead box (Fox) $3^{+}$regulatory $\mathrm{T}$ cells. C-c chemokine ligand 5 (CCL5) molecules in colorectal cancer cells may bind with CCR5 and initiate the CCL5/CCR5 signal pathway, which may recruit regulatory $\mathrm{T}$ cells to the tumor and increase the rate of tumor-induced anti-tumor $\mathrm{CD} 8^{+} \mathrm{T}$ cell death. Thus, the immune escape of colorectal cancer cells may occur through this pathway. In the present study, the expression of CCR5 increased significantly with increased malignancy of the epithelial cells $(\mathrm{P}<0.001)$, which was consistent with the results of Chang et al (8). The expression of CCR5 was not significantly different between the early cancer and adenoma groups ( $\mathrm{P}=0.176)$, which indicated that the expression of CCR5 was not altered significantly in early colorectal cancer, and that CCR5 may not be a major factor of the immune evasion in early colorectal cancer.

Although FasL was expressed highly in active immune cells such as CTL, studies have revealed that FasL is also expressed in colorectal cancer cells $(9,10)$. As active CTL expressed Fas is sensitive to Fas-mediated apoptosis, several studies have demonstrated that the expression of FasL in tumor cells may induce the apoptosis of tumor infiltrating lymphocytes $(9,10,20)$. Pryczynicz et al (11) reported that FasL was highly expressed in $70 \%$ of patients with colorectal cancer, and lowly expressed in $30 \%$ of patients with colorectal cancer, whilst all normal colorectal epithelial cells did not express FasL. Statistical analysis demonstrated that a high expression of FasL was associated with tumor angiogenesis and invasion. The present study suggested that the expression of FasL increased significantly with increased malignancy of the epithelial cells $(P<0.001)$, which was consistent with the results of aforementioned research. However, the expression of 
FasL was not significantly different between the early cancer and adenoma groups $(\mathrm{P}=0.605)$, which indicated that FasL may not be a major immune evasion-associated molecule of early colorectal cancer.

HLA-E is a type of atypical HLA class I molecule. Bossard et al (12) revealed that the HLA-E expressed in colorectal cancer cells may combine with the HLA-E receptor CD94/NKG2A expressed on the surface of CTL and NK cells, which contributed to the inhibition of the activity of the CTL and NK cells. Zhen et al (21) hypothesized that the overexpression of HLA-E in colorectal cancer cells was often associated with lower long-term disease-free survival rate and poor prognosis. Levy et al (13) demonstrated that HLA-E expression on the surface of colorectal cancer cell membranes could inhibit the cetuximab-mediated antibody-dependent cell-mediated cytotoxicity, which may promote the immune evasion of colorectal cancer cells. In the present study, the expression of HLA-E increased significantly as the malignancy of the epithelial cells increased $(\mathrm{P}<0.001)$. In addition, the expression of HLA-E increased significantly in the early cancer group $(\mathrm{P}<0.001)$ compared with the adenoma group, which suggested that the expression of HLA-E may serve a key role in the immune evasion in early colorectal cancer.

The sample size of the present study was relatively small and it was conducted in a single center. However, the immune evasion mechanisms of early colorectal cancer cells have been investigated.

The present study indicated that, the expression of Fas reduced significantly, whilst the expression of HLA-E increased significantly in early cancer group compared with adenoma group. It suggested that a loss of Fas expression and a high expression level of HLA-E may promote immune evasion in early colorectal cancer cells, which has not yet been investigated extensively. Thus, on the basis of the present study, additional studies with larger sample sizes that investigate the precise mechanisms of immune evasion in early colorectal cancer cells at the level of molecular transduction are required.

\section{Acknowledgements}

The present study would like to thank the Youth Scientific Research Project of Shanghai Health Bureau (grant no. 201344073). Furthermore, the present study acknowledges Professor Xu Fuxing, Dr Ji Danian and Dr Zhou Jun for reviewing the draft manuscript and providing feedback.

\section{References}

1. Torre LA, Bray F, Siegel RL, Ferlay J, Lortet-Tieulent J and Jemal A: Global cancer statistics. CA Cancer J Clin 65: 87-108, 2015.

2. Ren JS, Shi JF, Zhang HZ, Liu Q, Zhang YM, Zou SM, Zhang K and Dai M: Preliminary analysis of the colorectal cancer screening among urban populations in China, 2012-2013. Zhonghua Yu Fang Yi Xue Za Zhi 49: 441-443, 2015 (In Chinese).
3. Ge HL: Tumor immunity. In: Principles of immunology. Zhou GY (ed). Shanghai Scientific and Technical Publishers, Shanghai, pp279-294, 2007.

4. Bernal M, Ruiz-Cabello F, Concha A, Paschen A and Garrido F: Implication of the $\beta 2$-microglobulin gene in the generation of tumor escape phenotypes. Cancer Immunol Immunother 61: 1359-1371, 2012.

5. Kloor M, Michel S and von Knebel Doeberitz M: Immune evasion of microsatellite unstable colorectal cancers. Int J Cancer 127: 1001-1010, 2010.

6. Liu K: Role of apoptosis resistance in immune evasion and metastasis of colorectal cancer. World J Gastrointest Oncol 2: 399-406, 2010.

7. Sträter J, Hinz U, Hasel C, Bhanot U, Mechtersheimer G, Lehnert T and Möller P: Impaired CD95 expression predisposes for recurrence in curatively resected colon carcinoma: Clinical evidence for immunoselection and CD95L mediated control of minimal residual disease. Gut 54: 661-665, 2005.

8. Chang LY, Lin YC, Mahalingam J, Huang CT, Chen TW, Kang CW, Peng HM, Chu YY, Chiang JM, Dutta A, et al: Tumor-derived chemokine CCL5 enhances TGF- $\beta$-mediated killing of CD8(+) T cells in colon cancer by T-regulatory cells. Cancer Res 72: 1092-1102, 2012.

9. Houston AM, Michael-Robinson JM, Walsh MD, Cummings MC, Ryan AE, Lincoln D, Pandeya N, Jass JR, Radford-Smith GL and O'Connell J: The 'Fas counterattack' is not an active mode of tumor immune evasion in colorectal cancer with high-level microsatellite instability. Hum Pathol 39: 243-250, 2008.

10. Ryan AE, Shanahan F, O'Connell J and Houston AM: Fas ligand promotes tumor immune evasion of colon cancer in vivo. Cell Cycle 5: 246-249, 2006.

11. Pryczynicz A, Guzińska-Ustymowicz K and Kemona A: Fas/FasL expression in colorectal cancer. An immunohistochemical study. Folia Histochem Cytobiol 48: 425-429, 2010.

12. Bossard C, Bézieau S, Matysiak-Budnik T, Volteau C, Laboisse CL, Jotereau F and Mosnier JF: HLA-E/ $\beta 2$ microglobulin overexpression in colorectal cancer is associated with recruitment of inhibitory immune cells and tumor progression. Int J Cance 131: 855-863, 2012.

13. Levy EM, Sycz G, Arriaga JM, Barrio MM, von Euw EM, Morales SB, González M, Mordoh J and Bianchini M: Cetuximab-mediated cellular cytotoxicity is inhibited by HLA-E membrane expression in colon cancer cells. Innate Immun 15: 91-100, 2009.

14. Dukes CE: The surgical pathology of rectal cancer: President's Address. Proc R Soc Med 37: 131-144, 1944.

15. Sandel MH, Speetjens FM, Menon AG, Albertsson PA, Basse PH, Hokland M, Nagelkerke JF, Tollenaar RA, van de Velde CJ and Kuppen PJ: Natural killer cells infiltrating colorectal cancer and MHC class I expression. Mol Immunol 42: 541-546, 2005.

16. Menon AG, Tollenaar RA, van de Velde CJ, Putter H, Janssen-van Rhijn CM, Keijzer R, Fleuren GJ and Kuppen PJ: p53 and HLA class-I expression are not down-regulated in colorectal cancer liver metastases. Clin Exp Metastasis 21: 79-85, 2004.

17. Menon AG, Janssen-van Rhijn CM, Morreau H, Putter H, Tollenaar RA, van de Velde CJ, Fleuren GJ and Kuppen PJ: Immune system and prognosis in colorectal cancer: A detailed immunohistochemical analysis. Lab Invest 84: 493-501, 2004.

18. Watson NF, Ramage JM, Madjd Z, Spendlove I, Ellis IO, Scholefield JH and Durrant LG: Immunosurveillance is active in colorectal cancer as downregulation but not complete loss of MHC class I expression correlates with a poor prognosis. Int J Cancer 118: 6-10, 2006.

19. Yang D, Stewart TJ, Smith KK, Georgi D, Abrams SI and Liu K: Downregulation of IFN-gammaR in association with loss of Fas function is linked to tumor progression. Int J Cancer 122: 350-362, 2008

20. Krammer PH: CD95's deadly mission in the immune system. Nature 407: 789-795, 2000.

21. Zhen ZJ, Ling JY, Cai Y, Luo WB and He YJ: Impact of HLA-E gene polymorphism on HLA-E expression in tumor cells and prognosis in patients with stage III colorectal cancer. Med Oncol 30: 482, 2013. 